\title{
Actuación veterinaria en perros de trabajo en incidentes con opioides
}

\author{
Cique-Moya A. ${ }^{1}$
}

Sanid. mil. 2021; 77 (1): 40-44, ISSN: 1887-8571

\begin{abstract}
RESUMEN
Los perros de trabajo, mayormente los perros detectores de drogas y estupefacientes pueden resultar intoxicados con opioides. Fundamentalmente fentanilos, en el desarrollo de su actividad. En función de la posibilidad de penetración por diferentes vías, los animales contaminados suponen un riesgo para los dueños, los guías y los veterinarios que los manejen y traten. Lo cual, unido a su toxicidad hace necesario aplicar el tratamiento antidotal con naloxona de forma inmediata a la aparición de los signos y síntomas hasta la reversión de los mismos (de 1 a $4 \mathrm{mg}$ por $25 \mathrm{~kg}$ en función de la vía de administración). Razón por la cual, resulta prioritario no solo saber reconocer y tratar los síntomas de la intoxicación (triada por sobredosis de opioides), sino que resulta fundamental realizar una correcta evaluación de la situación, adoptar el adecuado nivel de protección física individual por parte del personal de intervención, así como la necesidad de integrar la capacidad de descontaminación en los procedimientos de intervención para gestionar este tipo de incidentes y así reducir el riesgo de transferencia de contaminación.
\end{abstract}

PALABRAS CLAVE: opioides, intoxicación, perros, naloxona, equipos cinológicos.

\section{Veterinary intervention in working dogs regarding opioid related incidents SUMMARY}

Working dogs, primarily narcotics detection dogs may be intoxicated with opioids. Mainly with fentanyl within the scope of the drug detection activities. Depending on the nature of the various routes of penetration, contaminated animals may pose a risk to owners, trainers and veterinarians whom are in charge of their treatment and care. Given its toxicity, immediate antidotal treatment with naloxone is required after the initial onset of symptoms until remission of those symptoms ( 1 to $4 \mathrm{mg}$ per $25 \mathrm{~kg}$ depending on the route of drug administration). Following the above, it is not only a priority to know how to recognize and treat the various symptoms of intoxication but to correctly evaluate the situation and assure proper adoption of adequate physical protection by the first responders as well as to integrate proper decontamination guidelines in the intervention procedures to better facilitate this type of incident management and reduce the risk of contamination.

KEY WORDS: opioids. Intoxication, dogs, naloxone, K9 teams.

\section{INTRODUCCIÓN Y OBJETIVOS}

Los animales de compañía no están exentos de sufrir intoxicaciones por drogas de abuso, ya sea con carácter accidental o provocado. En la mayoría de las ocasiones las vías de entrada de estas sustancias en el organismo son la oral y la respiratoria. Los perros detectores de drogas pueden tener más posibilidades de resultar intoxicados con sustancias controladas, como estupefacientes y psicotrópicos, en el desarrollo de su actividad. Estas sustancias, pueden provocar cambios temporales en la percepción, ánimo, estado de conciencia y comportamiento, con lo que aquellos que estén en contacto con ellos deben de extremar las precauciones en su manejo ${ }^{1-3}$.

${ }^{1}$ Coronel Veterinario. Jefatura Conjunta de Sanidad - Estado Mayor de la Defensa. Calle Vitruvio, 1, 28006 Madrid.

Dirección para correspondencia: Alberto Cique Moya - Coronel Veterinario. Jefatura Conjunta de Sanidad. Estado Mayor Conjunto. - Estado Mayor de la Defensa - Madrid. Calle Vitruvio, 1, 28006 Madrid. Tlf: 917455200 aciquemo@et.mde.es

Recibido: 22 de julio de 2020

Aceptado: 22 de diciembre de 2020

doi: $10.4321 /$ S1887-85712021000100007
Las intoxicaciones más frecuentes en animales domésticos, concretamente en los perros de trabajo en los Estados Unidos, son las causadas por los opioides, fundamentalmente los sintéticos, que son un componente o adulterante de la heroína ${ }^{4}$. En adición a esto, en Europa, aunque sin comparación posible con lo que sucede en Estados Unidos, en el año 2017 se realizaron más de 300 incautaciones de fentanilo y más de 600 de carfentanilo líquido y en polvo (mezclado o no con heroína) ${ }^{5,6}$, lo cual unido a que una de cada cinco personas que se someten a tratamiento por una adicción relacionada con el consumo de opioides declara que su principal problema no es la heroína, sino los opioides sintéticos genera que los animales puedan resultar afectados por estas sustancias, en el transcurso de una intervención ${ }^{7}$.

Un riesgo para los guías caninos, cuidadores y veterinarios que tratan con animales intoxicados es la posibilidad de resultar afectado debido a que algunas sustancias depositadas sobre la piel o equipo en forma pulverulenta o líquida pueden penetrar en el organismo por vía respiratoria, mucosas o a través de la piel provocando una intoxicación más o menos grave en función de la dosis absorbida. Este riesgo supone que el personal que los maneja debe de adoptar el adecuado nivel de protección física individual, respiratoria y corporal, para evitar resultar contaminado ${ }^{8,9}$. 


\section{Actuación veterinaria en perros de trabajo en incidentes con opioides}

Es importante que los guías estén formados para reconocer los signos y síntomas, así como los primeros auxilios a aplicar en este tipo de situaciones en tanto en cuanto los animales reciben asistencia veterinaria. Por otro lado, el veterinario debe realizar una anamnesis cuidadosa teniendo en cuenta el inicio los síntomas, la sustancia causante de la intoxicación y la cantidad a la que ha sido expuesto para así instaurar el tratamiento más adecuado, aunque esta información es difícil que sea completa en todos los casos. También sería importante integrar en los operativos de intervención de los equipos veterinarios protocolos para una adecuada gestión de los incidentes mediante la evaluación de las condiciones de la intervención y la instauración de los tratamientos de emergencia ${ }^{10}$.

El objetivo de esta revisión es poner en evidencia el riesgo de intoxicación de los perros de trabajo con opiáceos sintéticos y la necesidad de que los guías caninos y los veterinarios puedan reconocer y gestionar este tipo de incidentes aplicando el tratamiento antidotal adecuado adoptando el adecuado nivel de protección física individual al objeto de prevenir el riesgo de transferencia de la contaminación.

\section{OPIOIDES}

Se entiende por opioide como aquella sustancia endógena (endorfinas) o exógena (de origen sintético o semisintético), con actividad intrínseca y efectos análogos a los de la morfina. Los opioides actúan sobre los receptores $\mu(M u), \kappa($ Kappa), $\delta$ (delta) (OPRM, OPRK y OPRD) y ORL-1 (opioid receptor like-1). Estos receptores pertenecen a la familia de receptores acoplados a proteina $G i$; de esta manera, inhiben la adenilciclasa y canales de calcio dependientes de voltaje y estimulan canales de potasio y la fosfodiesterasa $C$. Al disminuir el AMPc intracelular, los opioides modulan la liberación de neurotransmisores como la sustancia $P, G A B A$, dopamina, acetilcolina y norepinefrina y ejercen una variedad de efectos dependiendo del tipo de receptor estimulado ${ }^{11}$. Pueden verse afectados, en función de la sustancia implicada, los receptores colinérgicos, adrenérgicos, serotoninérgicos y/o dopaminérgicos distribuidos por toda la economía orgánica.

Los opioides se clasifican de acuerdo con su actividad en: agonistas puros (morfina, heroína, petidina, fentanilos, metadona, etc.), agonistas-antagonistas (pentazocina, nalbufina, butorfanol, etc.) agonistas parciales (buprenorfina, etc.) y antagonistas (naloxona, naltrexona, etc.) ${ }^{12-14}$.

En relación con su farmacocinética los opioides se absorben bien tras su administración oral (la más frecuente en perros), por vía respiratoria-nasal (heroína, fentanilos, o buprenorfina) o por vía transdérmica (fentanilos). Se metabolizan en el hígado y se distribuyen por vía hemática por los pulmones, el bazo, el músculo esquelético, el sistema nervioso central y por la placenta; eliminándose finalmente por los riñones ${ }^{15}$.

En lo que concierne a su toxicidad, la heroína, el fentanilo y el carfentanilo son de 100 hasta 10.000 veces más potentes que la morfina. De ahí que al mezclarse se potencien sus efectos tóxicos, habiéndose establecido en perros que la dosis letal 50 (DL50) de la morfina está en $100 \mathrm{mg} / \mathrm{kg}$ por vía subcutánea o intravenosa, mientras que la dosis letal de heroína está en $25 \mathrm{mg} / \mathrm{kg}$ por vía subcutánea y la del fentanilo en $14 \mathrm{mg} / \mathrm{kg}^{16}$.

\section{SINTOMATOLOGÍA Y DIAGNÓSTICO}

El cuadro clínico puede iniciarse entre uno y treinta minutos tras la exposición en función de la vía de entrada y la dosis absorbida. Por esta razón, los animales de trabajo que hayan participado en un operativo deban de mantenerse en observación en la siguiente hora al objeto de comprobar si aparecen signos o síntomas de intoxicación, toda vez que en función de los síntomas que desarrollan resulta complicado diferenciar si es un opioide (heroína, cocaína, metanfetaminas, benzodiacepinas, marihuana, fentanilo o carfentanilo), un tóxico como el etilenglicol, un insecticida como la ivermectina, o incluso que los síntomas estén asociados a un estado hipoglucémico.

En función del receptor afectado y del tipo de opioide implicado los perros muestran, al igual que los seres humanos, lo que se denomina la «triada por sobredosis de opioides»: depresión respiratoria, miosis y pérdida de conciencia. Los animales pueden no responder a las órdenes de los guías, mostrarse indiferentes a la recompensa e inquietos o tener la mirada pérdida. Pudiendo, en función de la dosis absorbida, el tipo de sustancia implicada y el receptor al que se haya unido en mayor proporción, presentar signos de alteración del comportamiento, depresión, somnolencia, ataxia, vómitos, convulsiones, miosis, hipotensión, hipotermia, letargia, bradicardia, bradipnea y, en los casos más graves de intoxicación, depresión respiratoria, coma y muerte. Razón por la cual, una vez iniciados los síntomas se requiere asistencia veterinaria inmediata ya que, si no se administra el tratamiento $\mathrm{y}$, a pesar de que los animales son más resistentes que los seres humanos, puede que los síntomas no reviertan y el animal muera como consecuencia de una parada cardiorrespiratoria ${ }^{17}$.

El diagnóstico clínico se basa en la historia de exposición a los opioides (depresión respiratoria y signos del sistema nervioso central). Resultando muy útil para el diagnóstico, aún a pesar de no estar indicados para los perros, la determinación de niveles plasmáticos en sangre o de metabolitos en orina mediante kits comerciales de humana siempre que se sigan las indicaciones del fabricante ${ }^{18}$.

\section{TRATAMIENTO}

Ante la duda de exposición a opioides, sea cual sea la vía de entrada en el organismo, resulta prioritario administrar hidrocloruro de naloxona, la cual, al ser un derivado semisintético de la morfina (N-alil-nor-oximorfona), es un antagonista específico de los opioides que actúa competitivamente en los receptores opiáceos $^{19}$.

En caso de que se sospeche que el animal hubiera ingerido opioides, la administración de naloxona permite confirmar el diagnóstico al producirse la reversión de los síntomas. La administración de naloxona en perros debe instaurarse a la mayor brevedad posible tras la aparición de los síntomas a las siguientes dosis seriadas en el tiempo en función de la vía de administración y de la evolución del animal: Intravenosa o intraósea: 0,01 a $0,04 \mathrm{mg} / \mathrm{kg}$ (1 a $2 \mathrm{mg}$ por $25 \mathrm{~kg}$ ); Intramuscular: 0,04 a $0,16 \mathrm{mg} / \mathrm{kg}$ (2 a $4 \mathrm{mg}$ por $25 \mathrm{~kg}$ ); Intranasal: 2 a $4 \mathrm{mg}$ por $25 \mathrm{~kg}$. En caso de imposibilidad de acceso por otras vías se puede utilizar la vía sublingual a las dosis indicadas para la vía intranasal (mediante espray nasal, 
gotas nasales), describiéndose también en la literatura científica la administración subcutánea y rectal y para la vía sublingual mediante hisopos saturados con el fármaco ${ }^{20-25}$.

El Manual Merck establece que el tratamiento sintomático de las otras manifestaciones clínicas como puedan ser las reacciones disfóricas (vocalización, agitación, inquietud y excitación) se pueden tratar con diazepam u otras benzodiacepinas. Para el síndrome serotoninérgico (desorientación, rigidez muscular, agitación) inducida por algunos opioides, se puede administrar ciproheptadina $(1,1 \mathrm{mg} / \mathrm{kg}$, via oral o rectal) una o dos veces (con una separación de 6 a 8 horas) ${ }^{26,27}$.

Debido a que la vida media del organismo de la naloxona es menor que la de los opioides (fundamentalmente el carfentanilo), puede darse la circunstancia de que tras su administración, los animales muestren un agravamiento de los síntomas (renarcotización), que haga preciso administrar dosis seriadas de naloxona al objeto de mantener la consciencia y la respiración espontánea, toda vez que la naloxona tiene un amplio margen de seguridad y practicamente ninguna acción agonista. Motivo por el cual, se deben de mantener a los animales monitorizados de 6 a 12 horas después de la exposición. En este sentido, no hay estudios que describan los efectos de la administración de dosis seriadas en los perros en el tiempo a pesar de que se recomiende su uso ${ }^{28}$.
La administración de naloxona no está exenta de riesgos, provocando en función de la sensibilidad individual y la dosis administrada provocar un cuadro de perdida de conciencia, taquicardia, hipo o hipertensión, náuseas o vómitos entre los síntomas más importantes ${ }^{29}$.

\section{GESTIÓN DE INCIDENTES}

En la figura 1 se recoge el diagrama de flujo propuesto por Mitek y McMichael (2017) referido a la gestión de incidentes donde se sospeche la presencia de opioides en un operativo donde se hayan visto envueltos animales, ya sea de trabajo o domésticos ${ }^{30}$. La dosis propuesta de naloxona a administrar es la dosis mínima indicada por el Manual Merck de veterinaria. La naloxona es un medicamento de prescripción veterinaria, planteándose entonces la necesidad de establecer los procedimientos operativos para que los animales reciban tratamiento con naloxona y asistencia lo más rápidamente posible ${ }^{31}$.

En caso de ingestión resulta prioritario proceder a la descontaminación digestiva lo más rápidamente tras su consumo. Para lo cual, a los animales expuestos a opioides, incluso a aquellos que no muestran signos de intoxicación, se les debe administrar dosis repetidas de carbón activado ( 2 a $5 \mathrm{~g} / \mathrm{kg}$ cada 2 a 4 horas).

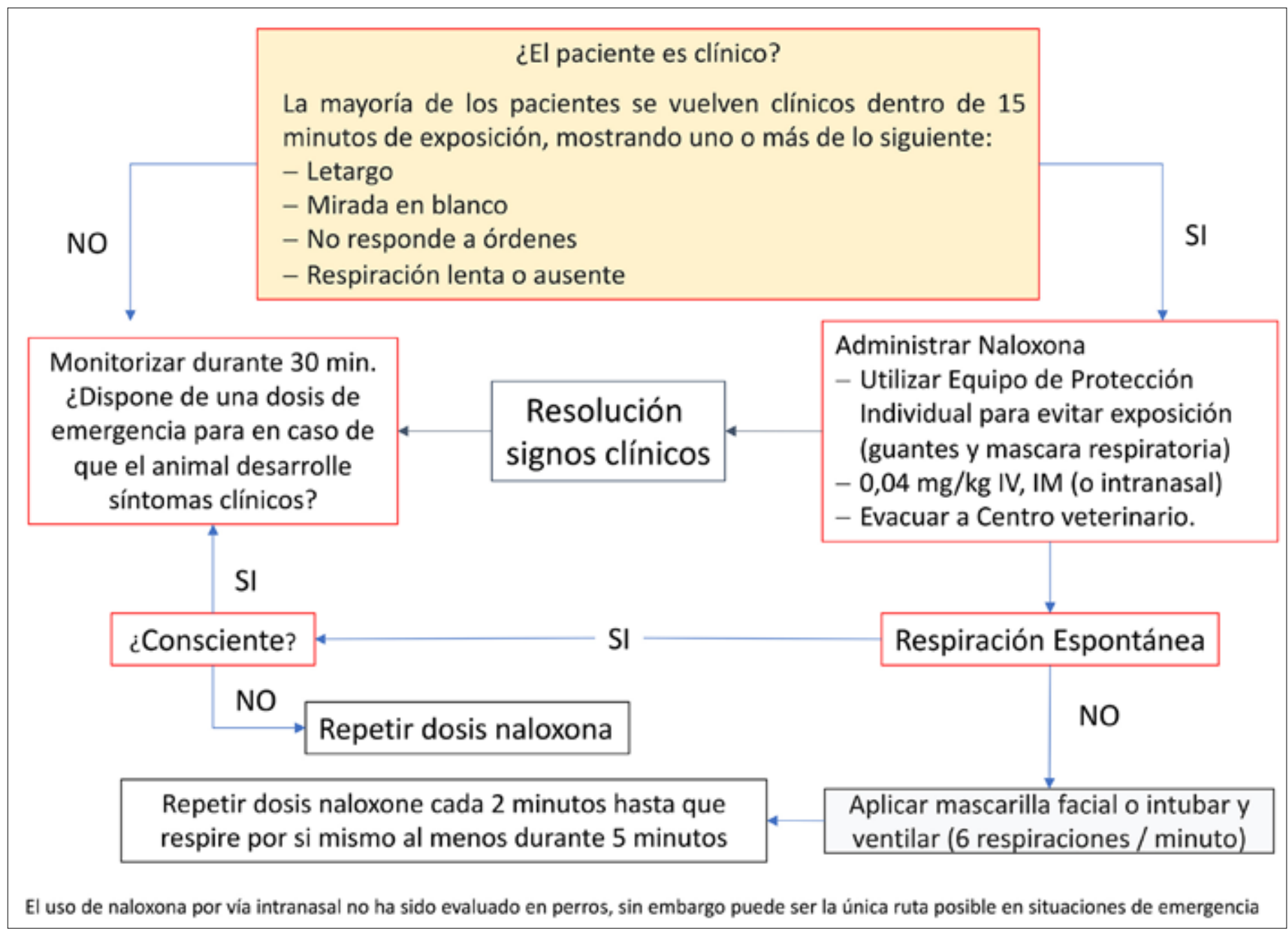

Figura 1. Diagrama de gestión de incidentes donde estén implicados opioides. 


\section{Actuación veterinaria en perros de trabajo en incidentes con opioides}

En caso de contaminación de la piel deben de ser descontaminados al objeto de evitar la penetración de éstos en el organismo, resultando suficiente el lavado con agua y jabón mediante un procedimiento reglado (de cabeza a cola y de cabeza a extremidades) $)^{32-34}$. En este sentido resulta fundamental desplegar la capacidad de descontaminación tanto para el personal como para los equipos cinológicos en operativos programados donde se tenga la certeza de que puedan estar implicados opioides ${ }^{35}$.

Al igual que en el hombre, resulta conveniente controlar la presión arterial, así como el ritmo, frecuencia cardiaca, mantener la vía aérea y asegurar una adecuada ventilación debido a los efectos depresores de los opioides sobre el sistema respiratorio. Debiéndose hacer hincapié en que la respiración boca a boca al animal está absolutamente contraindicada debido al riesgo de transferencia de la contaminación que puede resultar mortal para el hombre, recomendándose la necesidad de llevar guantes para manejar a los animales intoxicados. Esto es debido a que puede quedar restos pulverulentos en el cuerpo del animal y por tanto ser transferidos al guía/dueño/veterinario, lo cual conlleva que sea necesario adoptar el nivel de protección física adecuado ${ }^{36}$.

\section{NIVELES DE PROTECCIÓN FÍSICA INDIVIDUAL}

La posibilidad de transferencia de contaminación al personal que maneja los perros en incidentes donde se vean envueltos opioides hace necesario que éstos adopten el adecuado nivel de protección física individual, siendo prioritario retirar de la superficie de la ropa o de la piel cualquier tipo de contaminante, ya sea en forma líquida o en forma pulverulenta.

Resulta fundamental la concienciación del personal de intervención para que adopte comportamientos adecuados en este tipo de incidentes. Entre los que destaca realizar actividades de riesgo, como pueda ser comer y/o beber durante la intervención y nunca antes de quitarse el Equipo de Protección Individual (EPI) que hubiera utilizado o quitarse el uniforme en caso de que se detectara la presencia de sustancias pulverulentas y/o líquidas que se hubieran depositado sobre el equipo. Es necesario formar al personal para que utilice agua con jabón para retirar el contaminante de forma eficaz y evitar el uso de geles o toallitas desinfectantes con base alcohólica o embebidas con solución de hipoclorito sódico o cálcico ya que potencian la absorción de los opioides, fundamentalmente los fentanilos ${ }^{37}$

En relación con la seguridad asociada al nivel de protección física adoptado por el personal de intervención, hay que tener en cuenta que el EPI por si mismo no es suficiente para asegurar protección frente a los opioides. Es importante una sólida formación y entrenamiento (instrucción y adiestramiento) para aumentar la seguridad operativa del personal en la intervención, tanto en el reconocimiento de los signos y síntomas de intoxicación, como en la retirada normalizada del EPI esté o no integrada dentro del proceso de descontaminación y el posible destino final del equipo utilizado, ya sea en una posible reutilización o en una adecuada gestión de residuos ${ }^{38,39}$.

El personal de los equipos cinológicos y de los equipos veterinarios, ante la sospecha de que el perro haya resultado intoxicado con opioides, sobretodo con fentanilos, deberá adoptar el adecuado nivel de protección física individual. Los guantes de nitrilo, en condiciones normales, son suficientes para impedir la absorción cutánea. No obstante, en caso de que se hayan producido intervenciones en espacios cerrados muy contaminados se plantea la necesidad de utilizar guantes que confieran mayor protección (neopreno o butilo). Para la protección de las membranas mucosas y de los ojos se plantea el uso de pantallas faciales o máscaras faciales completas (fundamentalmente frente a las salpicaduras generadas durante la descontaminación). Resultando necesario para la protección respiratoria utilizar mascarillas tipo FFP2 o FFP3 o máscaras faciales, autoventiladas o no, completas con filtro P3. Para la protección corporal es suficiente utilizar, en función del puesto táctico, monos de categoría III, tipo 3 a $6^{40,41}$.

\section{CONCLUSIÓN}

La posibilidad de que los animales domésticos y los animales de trabajo resulten intoxicados por sustancias controladas como los opioides, es una realidad frente a la cual tanto los veterinarios como los guías caninos deben estar preparados no solo para reconocer y tratar los signos y síntomas de la intoxicación, sino que deben de ser capaces de operar con el adecuado nivel de protección física individual al objeto de evitar resultar contaminados debido a la transferencia de la contaminación. Lo cual conlleva la necesidad de introducir en los procedimientos operativos establecidos la capacidad de descontaminación no solo para el personal, sino también para los animales.

\section{BIBLIOGRAFÍA}

1. Howard-Azzeh M, Pearl DL, O'Sullivan TL, Berke O. The identification of risk factors contributing to accidental opioid poisonings in companion dogs using data from a North American poison control center (2006-2014). PLoS ONE 2020; 15(1): e227701. https://doi.org/10.1371/journal. pone.0227701.

2. Khan Safdar A. Toxicities from illicit and Abused Drugs. MSD Manual Veterinary Manual. 2019 (accedido 15/12/2019). Disponible en: https://www. msdvetmanual.com/toxicology/toxicities-from-human-drugs/toxicities-fromillicit-and-abused-drugs.

3. Kirkland T. Drug-sniffing K-9 are overdosing on opioids during routine searches: "This is a really big issue». 15/06/2018 (accedido 01/07/20). Disponible en: https://www.foxnews.com/us/drug-sniffing-k-9s-are-overdosing-on-opioids-during-routine-searches-this-is-a-really-big-issue.

4. National Institute on Drug Abuse. Opioid Overdose Crisis. enero 2019 (accedido 01/07/20). Disponible en: https://www.drugabuse.gov/drugs-abuse/ opioids/opioid-overdose-crisis

5. Sistema Español de Alerta Temprana - Delegación del Gobierno para el Plan Nacional sobre Drogas. Alerta Sobre Derivados del Fentanilo en Consumidores de Heroína. Sistema Español de Alerta Temprana - Delegación del Gobierno para el Plan Nacional sobre Drogas 24/05/18 (accedido 01/07/20). Disponible en: http://www.pnsd.mscbs.gob.es/profesionales/sistemasAlerta/ pdf/ALERTA_DERIVADOS_FENTANILO_CONSUMIDORES_HEROINA_29052018_def.pdf.

6. European Monitoring Centre for Drugs and Drug Addiction. EMCDDAEuropol Joint Report on a new psychoactive substance: methyl 1-(2-phenylethyl)-4-[phenyl(propanoyl)amino]piperidine-4-carboxylate (carfentanil). Publications Office of the European Union, Luxembourg. 2017 (accedido 01/07/20). Disponible en: https://www.emcdda.europa.eu/publications/joint-reports/carfentanil_en.

7. Observatorio Europeo de las Drogas y las Toxicomanías. Informe Europeo sobre Drogas 2019. Tendencias y novedades. Oficina de Publicaciones de la Unión Europea. Luxemburgo. (accedido 01/07/20). 
Disponible en: http://www.emcdda.europa.eu/system/files/publications/11364/20191724_TDAT19001ESN_PDF.pdf.

8. Department of Homeland Security. Protecting First Responders on the Frontlines of the Fentanyl Epidemic. Proceedings from the 2017 Working Meeting. Department of Homeland Security - Countering Weapons of Mass Destruction - Office of Health Affairs. September 6-7, 2017. 01/05/18 (accedido 01/07/20). Disponible en: https://www.dhs.gov/sites/default/files/publications/ochco-dhs-proceedings-from-fentanyl-working-meeting-20180501.pdf.

9. Cima G. Synthetic opioids put police dogs at risk. American Veterinary Medical Association 18.01.18 (accedido 01/07/20). Disponible en: https://www. avma.org/javma-news/2018-02-01/synthetic-opioids-put-police-dogs-risk.

10. Brown AJ, Mandell DC. Illicit drugs. En: Small Animal Critical Care Medicine. Chapter 81. 2009: 342-345.

11. Nava-Mesa MO, Tellez-Arevalo A, Rojas-Kozhakin D, Calderón-Ospina CA. Usos terapéuticos potenciales de los antagonistas opioides: fisiopatología y evidencia preclínica. Rev. Colomb. Cienc. Quim. Farm. 2015; 44(3): 322-358.

12. Valdivielso Serna A. Dolor agudo, analgesia y sedación en el niño (IIIa): Farmacocinética y farmacodinamia de los analgésicos opioides. Anales Españoles de Pediatria 1998; 48(4): 429-440.

13. Junta de Andalucía. Definición y descripción de las propiedades de algunos opiáceos y opioides. Consejería para la Igualdad y Bienestar social (accedido 01/07/20). Disponible en: https://www.easp.es/pepsa/ estudios $+\mathrm{y}+$ documentos/monografiaopiodes.htm.

14. Muriel C, Santos J, Sánchez-Montero FJ. Farmacología de los analgésicos opiáceos. Máster del dolor - módulo 7. Cátedra extraordinaria del Dolor - Fundación Grunenthal (accedido 01/07/20). Disponible en: http://www.catedradeldolor.com/PDFs/Cursos/Tema\%207.pdf.

15. Schmiedt Chad W. Accidental prehension and suspected transmucosal or oral absorption of fentanyl from a transdermal patch in a dog. Veterinary Anaesthesia and Analgesia 2007; 34(1): 70-73 https://doi.org/10.1111/j.14672995,2006,00302.x.

16. Bischoff K. Toxicity of drugs of abuse. En: Veterinary Toxicology, Edited by Ramesh C. Gupta Academic Press 2007: 391-410.

17. Malouin A, Boller M. Sedatives, Muscle Relaxants, and Opioids Toxicity. En: Small Animal Critical Care Medicine. Chapter 83. 2009: 350-356.

18. Khan Safdar A. Toxicities from Illicit and Abused Drugs (accedido 01/07/20). Disponible en: https://www.msdvetmanual.com/toxicology/toxicities-fromhuman-drugs/toxicities-from-illicit-and-abused-drugs.

19. Agencia Española del Medicamento y de los Productos Sanitarios. Ficha técnica Naloxona B. Braun $0,4 \mathrm{Mg} / \mathrm{Ml}$ solución inyectable y para perfusión. Marzo 2018 (accedido 01/07/20). Disponible en: https://cima.aemps.es/cima/ pdfs/es/ft/69533/FT_69533.pdf.

20. Goldfrank, LR Weisman, RS. Opioids. En: Toxicologic Emergencies, Goldfrank, LR, Flomenbaum, NE, Lewin, NA, Weisman, RS, Howland, MA and Hoffman, RS (eds.), Appleton and Lange, Norwalk, C 1994: 847-862.

21. Poppenga RH. Illicit Drug Intoxications of Small Animals (accedido 01/07/20). Disponible en: https://www.vin.com/apputil/content/defaultadv1. aspx?pId $=11131 \&$ catId $=29210 \&$ id $=3844041$.

22. Mieke Robertson T, Hendey Gregory W, Stroh G, Shal M. La administración intranasal de naloxona es una alternativa viable a la administración intrevanosa de naloxona en los pacientes con sobredosis de opiáceos atendidos en el contexto prehospitalario. Prehospital EMergency CAre (Ed. Esp.), 2010; $3(1): 31-35$.

23. College of Veterinary Medicine. Emergency Protocol: Canine Opioid Exposure or Suspected Exposure. University of Illinois at Urbana-Champaing 14.03.17 (accedido 01/07/20). Disponible en: https://vetmed.illinois.edu/wpcontent/uploads/2017/04/opioid-emergency-protocol.pdf.

24. Copland VS, Haskins SC,Patz J. Naloxone reversal of oxymorphone effects in dogs Am J Vet Res.1989 Nov; 50(11): 1854-1858.

25. Gaynor JS. Intranasal Use of Naloxone in Working Canines. September 2016 http://usarveterinarygroup.org/usarvet/wp-content/uploads/2016/09/ Narcan_IN_for_Work_Dogs_OD.pdf.
26. Khan Safdar A. Toxicities from Illicit and Abused Drugs. Aug 2014 (accedido 10.05.20). Disponible en: https://www.msdvetmanual.com/toxicology/ toxicities-from-human-drugs/toxicities-from-illicit-and-abused-drugs.

27. Lobo M, Díaz MJ, García J, Frejo MT. Estudio piloto sobre la prescripción en situaciones excepcionales. Uso de medicamentos de humana en medicina veterinaria. Clin. Vet. Peq. Anim, 2011; 31(3): 143-149.

28. Brice-Saddler M. Jake the narcotics dog went into a prison to sniff for drugs. What he found killed him» The Washington Post (edición online) 23.07.19 (accedido 25.11.19). Disponible en: https://www.washingtonpost.com/science/2019/07/23/jake-narcotics-k-died-after-sniffing-synthetic-weed-an-alabama-prison/.

29. Organización Mundial de la Salud. Manejo del abuso de sustancias Información sobre la sobredosis de opioides. noviembre de 2014 (accedido 01/07/20). Disponible en: https://www.who.int/substance_abuse/ information-sheet/es/.

30. Mitek A, McMichael M. Emergency Protocol: Canine Opioid Exposure or Suspected Exposure Veterinary Medicine at Illinois 14.04.17 (accedido 30.06.20). Disponible en: https://vetmed.illinois.edu/wp-content/ uploads/2017/04/opioid-emergency-protocol.pdf.

31. College of Veterinary Medicine, Overdose in Working Dogs: Full Video Script. University of Carolina at Urbana-Champaign 14.05.17 (Accedido 01/07/20). Disponible en: https://vetmed.illinois.edu/overdose-working-dogsscript/.

32. Ayuso E. Urgencias Toxicológicas 13.04 .18 (Accedido 25/11/19). Disponible en: http://www.colvema.org/WV_descargas/intoxicacionesAMVAC-13042007193146.pdf.

33. Daza MA, Ayuso E. Intoxicaciones más frecuentes en pequeños animales. AVEPA, 2004: 24(4) 231-239.

34. Cique Moya A. Asistencia veterinaria en incidentes químicos» Profesión veterinaria 2018; 23(91): 76-88.

35. Alberta Health Services, Interim Guidance For First Responders: Opioid Misuse. Emergency Medical Services 01/17 (accedido 01/07/20). Disponible en: https://www.albertamfr.ca/data/documents/OPIOID_MISUSE_INTERIM_GUIDANCE_v.3.0.pdf.

36. Loos D. Police Dogs at Risk: Symptoms and Treatment of Opioid Exposure in Canine Officers 13.04.18 (Accedido 25/11/19). Disponible en: https://www. lexipol.com/resources/blog/police-dogs-risk-symptoms-treatment-opioidexposure-canine-officers/.

37. Office of Health Affairs. Protecting First Responders on the Frontlines of the Fentanyl Epidemic Proceedings from the 2017 Fentanyl Working Meeting. Department of Homeland Security Countering Weapons of Mass Destruction 01.05.18 (accedido 01/07/20). Disponible en: https://www.dhs.gov/sites/ default/files/publications/ochco-dhs-proceedings-from-fentanyl-workingmeeting-20180501.pdf.

38. Seal K, Grobelna A. Personal protective equipment for health care workers exposed to opioids: clinical effectiveness and guidelines. Ottawa: CADTH; (CADTH rapid response report: reference list). 2017 Sep. 08/09/17 (accedido 01/07/20). Disponible en: https://cadth.ca/sites/default/files/pdf/htis/2017/ RA0926\%20PPE $\% 20$ for $\% 20$ Opioid $\% 20$ Exposure $\% 20$ Final.pdf.

39. The Interagency Board. Recommendations on Selection and Use of Personal Protective Equipment and Decontamination Products for First Responders Against Exposure Hazards to Synthetic Opioids, Including Fentanyl and Fentanyl Analogues 08/17 (accedido 01/07/20). Disponible en: https://www. hsdl.org/?abstract\&did=803191.

40. American College of Medical Toxicology, American Academy of Clinical Toxicology «ACMT and AACT Position Statement: Preventing Occupational Fentanyl and Fentanyl Analog Exposure to Emergency Responders (Accedido 01/07/20). Disponible en: https://www.acmt.net/_Library/Positions/ Fentanyl_PPE_Emergency_Responders_.pdf.

41. 3M. Information to Help With Fentanyl Personal Protective Equipment Selection 3M (Accedido 01/07/20). Disponible en: https://multimedia.3m. com/mws/media/1458872O/fentanyl-white-paperus.pdf. 